\title{
A deep learning framework for imputing missing values in
}

\section{genomic data}

Yeping Lina Qiu ${ }^{1,2}$, Hong Zheng ${ }^{2}$, Olivier Gevaert ${ }^{2,3, *}$

${ }^{1}$ Department of Electrical Engineering, Stanford University, Stanford, CA, USA

${ }^{2}$ Medicine - Center for Biomedical Informatics Research, Stanford University, Stanford, CA

USA

${ }^{3}$ Biomedical Data Science, Stanford University, Stanford, CA, USA

* To whom correspondence should be addressed: ogevaert@stanford.edu 


\section{Abstract}

Motivation: The presence of missing values is a frequent problem encountered in genomic data analysis. Lost data can be an obstacle to downstream analyses that require complete data matrices. State-of-the-art imputation techniques including Singular Value Decomposition (SVD) and K-Nearest Neighbors (KNN) based methods usually achieve good performances, but are computationally expensive especially for large datasets such as those involved in pan-cancer analysis.

Results: This study describes a new method: a denoising autoencoder with partial loss (DAPL) as a deep learning based alternative for data imputation. Results on pan-cancer gene expression data and DNA methylation data from over 11,000 samples demonstrate significant improvement over standard denoising autoencoder for both data missing-at-random cases with a range of missing percentages, and missing-not-at-random cases based on expression level and GCcontent. We discuss the advantages of DAPL over traditional imputation methods and show that it achieves comparable or better performance with less computational burden.

Availability: https://github.com/gevaertlab/DAPL

Contact: ogevaert@stanford.edu 


\section{Introduction}

The Cancer Genome Atlas (TCGA) project has provided researchers a rich database to study the molecular basis of cancer (Cancer Genome Atlas Research, et al., 2013). Pan-cancer analyses enable a better understanding of the genomic commonalities and differences across tumor types and subtypes, which makes it possible to extend diagnosis and treatment from one cancer type to another with a similar genomic profile (Cline, et al., 2013). RNA sequencing data and DNA methylation data have been widely investigated in pan-cancer analyses to identify cancer driver genes or biomarkers (Byron, et al., 2016; Gevaert, et al., 2015; Kulis and Esteller, 2010; Litovkin, et al., 2015; Tomczak, et al., 2015). Many such studies on pan-cancer genomics require complete datasets (Champion, et al., 2018). However, missing values are frequently present in these data due to various reasons including low resolution, missing probes, and artifacts (Baghfalaki, et al., 2016; Libbrecht and Noble, 2015). Therefore, practical methods to handle missing data in pan-cancer genomic datasets are needed for effective downstream analyses.

One way to complete the data matrices is to ignore missing values by removing the entire feature if any of the samples has a missing value in that feature, but this is usually not a good strategy as the feature may contain useful information for other samples. The most preferable way to handle missing data is to impute their values in the pre-processing step. Many approaches have been proposed for this purpose, including replacement using average values, estimation using weighted K-nearest neighbor (KNN) method (Faisal and Tutz, 2017; Troyanskaya, et al., 2001), and estimation using singular value decomposition (SVD) based methods (Troyanskaya, et al., 2001). In recent years, a branch of machine learning which emerged based on big data and 
deep artificial neural network architectures, usually referred to as deep learning, has advanced rapidly and shown great potential for applications in bioinformatics (Min, et al., 2017). Deep learning has been applied in areas including genomics studies (Chen, et al., 2016; Leung, et al., 2014), biomedical imaging (Chen, et al., 2016), and biomedical signal processing (Wulsin, et al., 2011). Deep learning based methods have also been proposed to solve the missing data problems in various contexts and shown promising results (Beaulieu-Jones and Moore, 2017; Jaques, et al., 2018; Vincent, et al., 2008). Among the competitor algorithms for genomic data imputation, KNN and SVD are still considered the de facto standard because they can be easily implemented and have been reported to achieve best performances in missing value estimation problems (Aghdam, et al., 2017). However, when applied to very large datasets, KNN and SVD have severe drawbacks in computational overhead. Both methods are not model based and hence require significant computation at evaluation time, which makes them less practical in largescale genomic data imputation.

In this study, we propose to leverage the denoising autoencoder (DAE) structure to predict missing values in pan-cancer genomic analysis. We demonstrate that by using a denoising autoencoder with partial loss (DAPL), it is possible to achieve significant improvement over a standard denoising autoencoder. We also show that the performance is on a par with the most state-of-the-art competitor algorithms while requiring less computational cost at evaluation time, for both data missing-at-random cases across a range of missing percentages, and missing-not-at-random cases based on expression level and GC-content. 


\section{Materials and Methods}

\section{Datasets}

We used the pan-cancer RNA sequencing data and DNA methylation data from TCGA. We preprocessed the raw RNA sequencing data by removing the genes with NA values, and doing a $\log$ transformation followed by z-score transformation. The resulting matrix has 11069 samples and 17175 genes. We used the pan-cancer DNA methylation data to validate the algorithm on a larger and wider (feature dimension $>>$ sample size) dataset. The validation data was processed in the same way as the RNA sequencing data, which resulted in a matrix with 9664 samples and 269273 features (CpG sites).

\section{Autoencoder}

An autoencoder is an unsupervised deep neural network that is trained to reconstruct an input $X$ by learning a function $h_{w, b}(X) \approx X$. This is done by minimizing the loss function between the input $X$ and the network's output $X^{\prime}: L\left(X, X^{\prime}\right)$. The most common loss function is the root mean squared error:

$$
L\left(X, X^{\prime}\right)=\sqrt{\left\|X-X^{\prime}\right\|^{2}}
$$

An autoencoder consists of an encoder and a decoder. The encoder transforms the input to a latent representation, often such that the latent representation is in a much smaller dimension than the input (Ballard, 1987). The decoder then maps the latent embedding to the reconstruction of $X$. An autoencoder is often used as a dimensional reduction technique to learn useful representation of data (Sakurada and Yairi, 2014). 
A denoising autoencoder (DAE) aims to recover the noise-free, original input through deep networks given a noisy input (Vincent, et al., 2008). In each iteration of training, noise is added to the input $X$ to obtain $\hat{X}$. The loss is computed between the original $X$ and the reconstructed $\hat{X}^{\prime}$.

\section{Denoising autoencoder with partial loss}

When the added noise to DAE is in the form of masking noise, i.e., a random fraction of the input is set to 0, the DAE can be trained to estimate missing values (Vincent, et al., 2010). The $0 \mathrm{~s}$ in the training data simulate the missing elements and the reconstructed noise-free data will fill those positions with estimated values. However, a standard DAE aims to reproduce the whole dataset without focusing on the missing elements, while some of the other state-of-the-art imputing methods, such as SVD, focus on learning the missing elements iteratively. As a result, the performance of a standard DAE in estimating missing values is not on par with SVD, or KNN based methods.

To ameliorate this problem, we implemented a denoising autoencoder with partial loss (DAPL) to focus the neural network's learning on the missing positions. We minimized the root mean square error (RMSE) loss only on the missing values between the original input $X$, and the reconstructed $\hat{X}^{\prime}$ :

$$
L\left(X, X^{\prime}\right)=\sqrt{\left\|X_{[\text {missing }]}-\hat{X}_{[\text {missing }]}^{\prime}\right\|^{2}}
$$




\section{Missing data simulations}

With the TCGA RNA sequencing dataset, we only used the features that have values for every sample to construct a ground truth complete data. Then the complete data was split $60 / 15 / 25 \%$ for training, validation, and testing.

We simulated two missingness scenarios: at random and not at random. In the missing at random case, missing values in the testing data are in this form: a fixed fraction of elements at random positions were masked by setting their values to NA. We experimented with a range of missing fraction: $5 \%, 10 \%, 30 \%, 50 \%$ and $80 \%$. We artificially introduced missingness to the complete training data by masking certain values. The autoencoder was initially trained such that the training data was masked to have the same fraction of random missingness as the testing data it was applied on. Then it was also trained on training data masked at different fraction of missingness than the testing data for comparisons. We then computed the RMSE error between the test ground truth and the imputed values. In the missing not at random case, we implemented two scenarios for missing. The first one was based on gene expression level (Conesa, et al., 2016). We tested on cases where a random half of the genes with expression level at the lowest $5 \%$ or $10 \%$ are missing. The autoencoder was trained on the same simulated scenario, where a random half of the genes with expression level at the lowest $5 \%$ or $10 \%$ were masked as missing during training. The second scenario was based on GC content, the percentage of nitrogenous bases on a RNA fragment that are either guanine $(\mathrm{G})$ or cytosine $(\mathrm{C})$. Too high or too low $\mathrm{GC}$ content influences RNA sequencing coverage, and potentially results in missing values (Chen, et al., 2013). We tested on cases where a random half of the genes with GC-content at the highest $5 \%$ or $10 \%$ are missing. Similarly, training was done on the same simulated scenarios. 


\section{Evaluation methods}

The model hyper parameters we tested include number of hidden layers, node size and dropout. We evaluated twelve autoencoder structures including three different number of layers (Figure 1, Table 1): 1) three layers with a layer-wise size reduction factor of 2, 4, 8 and 16-folds; 2) five layers with a layer-wise size reduction factor of 2, 3, 5, 8 and 10-folds; 3) seven layers with a layer-wise size reduction factor of 2, 3 and 4 -folds. Layer-wise size reduction factor is the amount of reduction in the number of nodes in one layer of an autoencoder compared to its previous layer in the encoder portion. For example, a reduction factor of two means one layer has approximately half of the number of nodes than its previous layer. Due to the symmetric architecture of autoencoders, the reduction factor in the encoder is the same as the layer-wise increase factor in the decoder. We will refer to this number as the layer-wise reduction factor. Dropout is a regularization technique to reduce autoencoder overfitting (Srivastava, et al., 2014). At training stage, individual units were dropped out of the network with a probability called dropout rate.

To evaluate denoising autoencoder with partial loss, we compared it to the other most commonly used missing value estimation methods: KNN method, SVD based method, standard DAE without loss modification, and column average imputation. KNN selects K number of samples which are most similar to the target sample with a missing gene based on Euclidean distance, and which all have values present in that gene. Imputation is a weighted average of the values of that gene in those $\mathrm{K}$ samples. We chose $\mathrm{K}=10$ in our evaluations based on a study which reported that $\mathrm{K}$ in the range of 10-25 gave the best imputation results (Troyanskaya, et al., 2001). SVD method decomposes the data matrix to the linear combination of eigengenes and 
corresponding coefficients. Genes are regressed against K most significant eigengenes, during which process the missing genes are not used (Hastie, et al., 1999). The obtained coefficients are linearly multiplied by eigengenes to get a reconstruction with missing genes filled. This process is repeated until the total change in the matrix reaches a certain threshold. The reconstruction performance of SVD depends on the number of eigengenes selected for regression, and we tested a range of values to determine the optimal K for SVD performance. Standard DAE was implemented as described previously, with loss defined over the entire data matrix. Column average imputation estimated the missing gene by taking the average of the gene in all samples where that gene was present.

We evaluated each test sample and computed RMSE error of the missing values in that sample

$$
R M S E=\sqrt{\sum_{i=1}^{n_{m i s s i n g}}\left(x_{i}-x_{i}^{\prime}\right)^{2} / n_{m i s s i n g}}
$$

Where $x_{i}$ is the element in the ground truth, and is $x_{i}^{\prime}$ is the element in the reconstructed data. Then we compared the RMSE distribution of the test samples for each of the method. Since SVD and KNN need to compute each time a new sample is tested, it is computationally expensive to evaluate all the test data. We randomly chose 100 samples from the test set for a fair comparison of all methods.

The method was validated on the TCGA pan-cancer DNA methylation data. Deep learning methods are usually faced with the challenges of severe overfitting and high-variance gradients when processing datasets that have a small sample size but high feature dimension 
(Liu, et al., 2017). We chose the methylation data where feature dimension is far greater than sample size to validate our algorithm's robustness and applicability in real world problems. 5\%, $30 \%$ and $80 \%$ random missing were tested on a five layers autoencoder which has a layer-wise size reduction of 100-fold with bottleneck size of 800 . Since it requires large computation resource to perform competitor algorithms on this large dataset, we did not run analysis on other random missing percentages and missing not-at-random cases due to hardware limitations.

Table 1. Autoencoder with Partial Loss (DAPL) structural variations

\begin{tabular}{|l|l|l|}
\hline $\begin{array}{l}\text { Layer } \\
\text { Number }\end{array}$ & $\begin{array}{l}\text { Reduction } \\
\text { Factor }\end{array}$ & $\begin{array}{l}\text { Bottleneck } \\
\text { Size }\end{array}$ \\
\hline \multirow{5}{*}{3 Layers } & 2 & 8000 \\
\cline { 2 - 3 } & 4 & 4000 \\
\cline { 2 - 3 } & 8 & 2000 \\
\cline { 2 - 3 } & 16 & 1000 \\
\hline \multirow{5}{*}{ L Layers } & 2 & 4500 \\
\cline { 2 - 3 } & 3 & 2000 \\
\cline { 2 - 3 } & 5 & 800 \\
\cline { 2 - 3 } & 8 & 250 \\
\cline { 2 - 3 } & 10 & 170 \\
\hline 7 Layers & 2 & 800 \\
\cline { 2 - 3 } & 3 & 400 \\
\cline { 2 - 3 } & 4 & 200 \\
\hline
\end{tabular}


(a)

Input dimension: $17176 \quad$ Output dimension: 17176

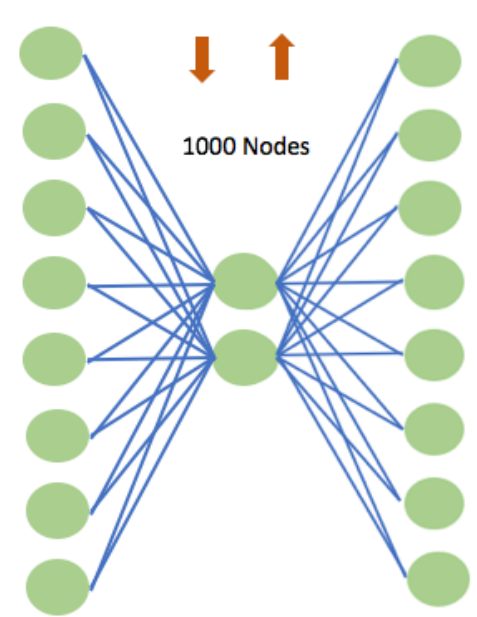

(b)

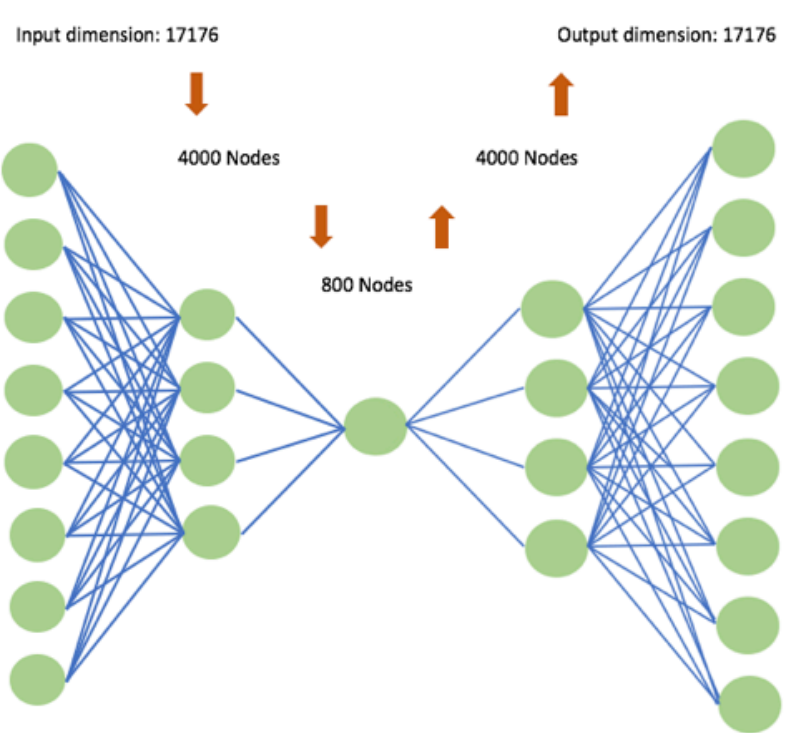

(c)

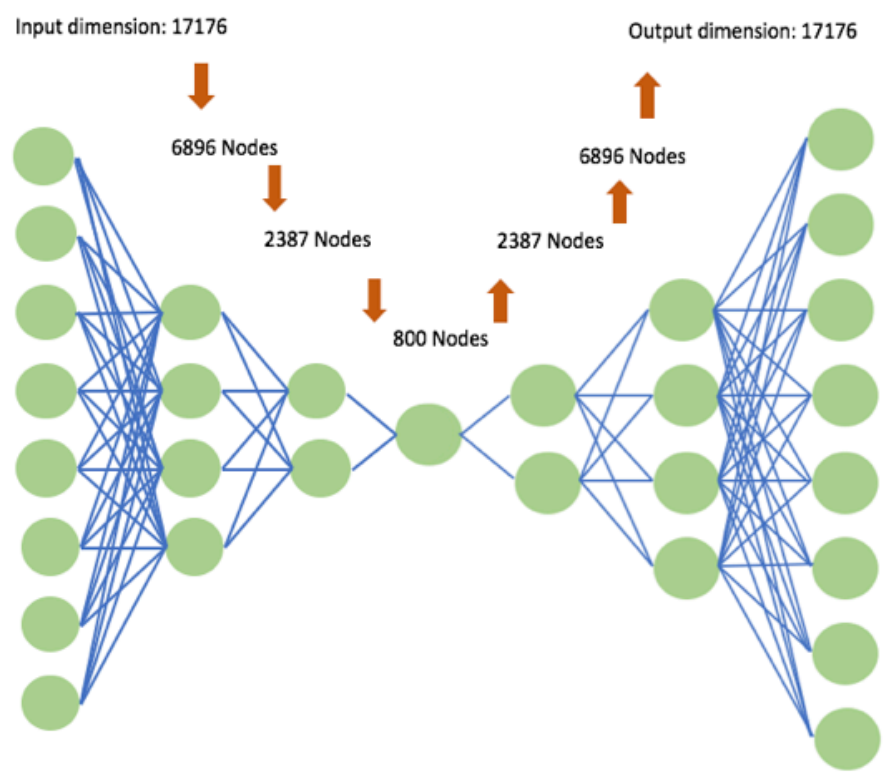

Figure 1. Schematics showing (a) a three layer autoencoder with bottleneck size of 1000. (b) a five layer autoencoders with bottleneck size of 800 , and (c) a seven layer autoencoders autoencoder with bottleneck size of 800 


\section{Results}

\section{DAPL outperforms DAE}

We compared the proposed AE models for different missingness scenarios and also evaluated different autoencoder structures. First we tested missing at random cases at varying percentage (Figure 2). $5 \%, 10 \%, 30 \%, 50 \%$, and $80 \%$ random elements in the data were masked respectively, and models were compared on the reconstruction root mean square errors (RMSE). Using a five hidden layers with 800 node bottleneck autoencoder structure, DAPL achieves significant improvement over the standard DAE for the comparisons between DAE and DAPL in all missing percentages (Wilcoxon rank sum test, P-value $<0.001$ ).

To determine the optimal number of eigengenes K used for regression in the SVD method, we experimented with $1 \%, 2.5 \%, 5 \%$, and $10 \%$ of the eigengenes and concluded that $2.5 \%-5 \%$ gives the best imputation results. The comparisons were done with $\mathrm{K}=2.5 \%$ of eigengenes. DAPL reaches an average error very close to the best performing SVD. 10-KNN method gives worse average error than SVD and DAPL, but slightly better than a standard denoising autoencoder without loss modification. Column average results in worst imputation performance among all methods considered. The situation does not change with changing noise levels.

We tested several dropout rates and found that dropout applied to hidden layers did not improve imputation performance. We also tested a different way of masking at training stage: we randomly selected a fixed fraction of columns in the whole data set, and set the entire columns to zero. We found it did not improve the imputation performance of DAPL. 


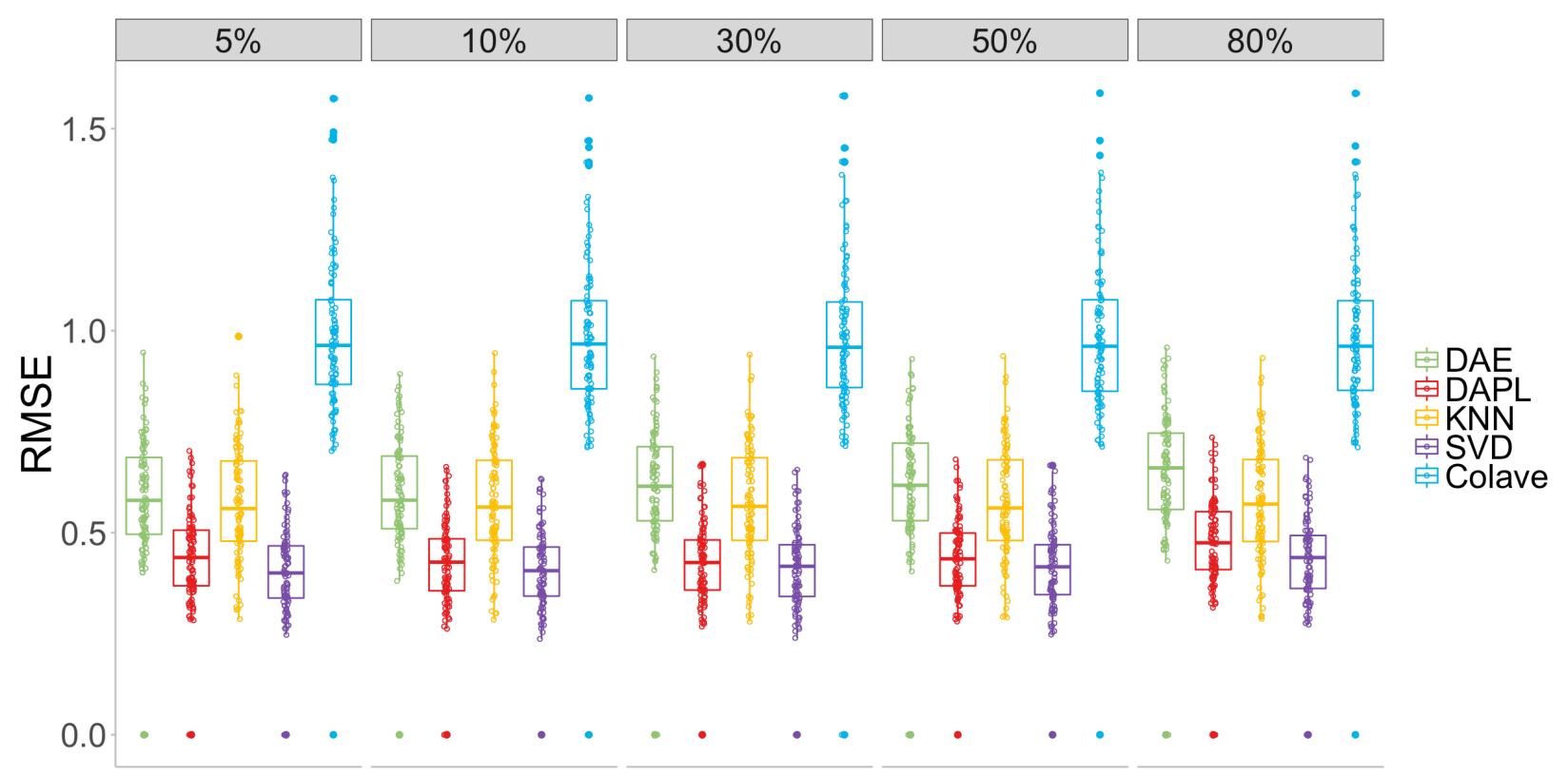

Figure 2. Comparisons of imputation methods measured in root mean square error (RMSE) for missing at random cases. From left to right: testing is done at 5\%,10\%, 30\%, 50\%, and 80\% random missingness. The method used from left to right: standard denoising autoencoder (DAE), denoising autoencoder with partial loss (DAPL), 10 nearest neighbor method (KNN), iterative singular value decomposition (SVD), and column average estimation (Colave).

\section{Optimal training for missing at random}

Next, we investigated the effect of different masking percentages during training of DAPL for predicting at specific missing levels. For each test case from the five missingness levels, DAPL was trained on all masking levels to find out which training scheme gives the best performance. Our results show that if training is done on the same simulated scenario as the testing case, i.e., the same level of missingness in testing is introduced during training, the performance is better than if training is done on a different scenario (Figure 3). However, it is also shown that when the testing missingness level is smaller than $50 \%$, training at $30 \%$ masked achieved reasonable performance at all levels. 

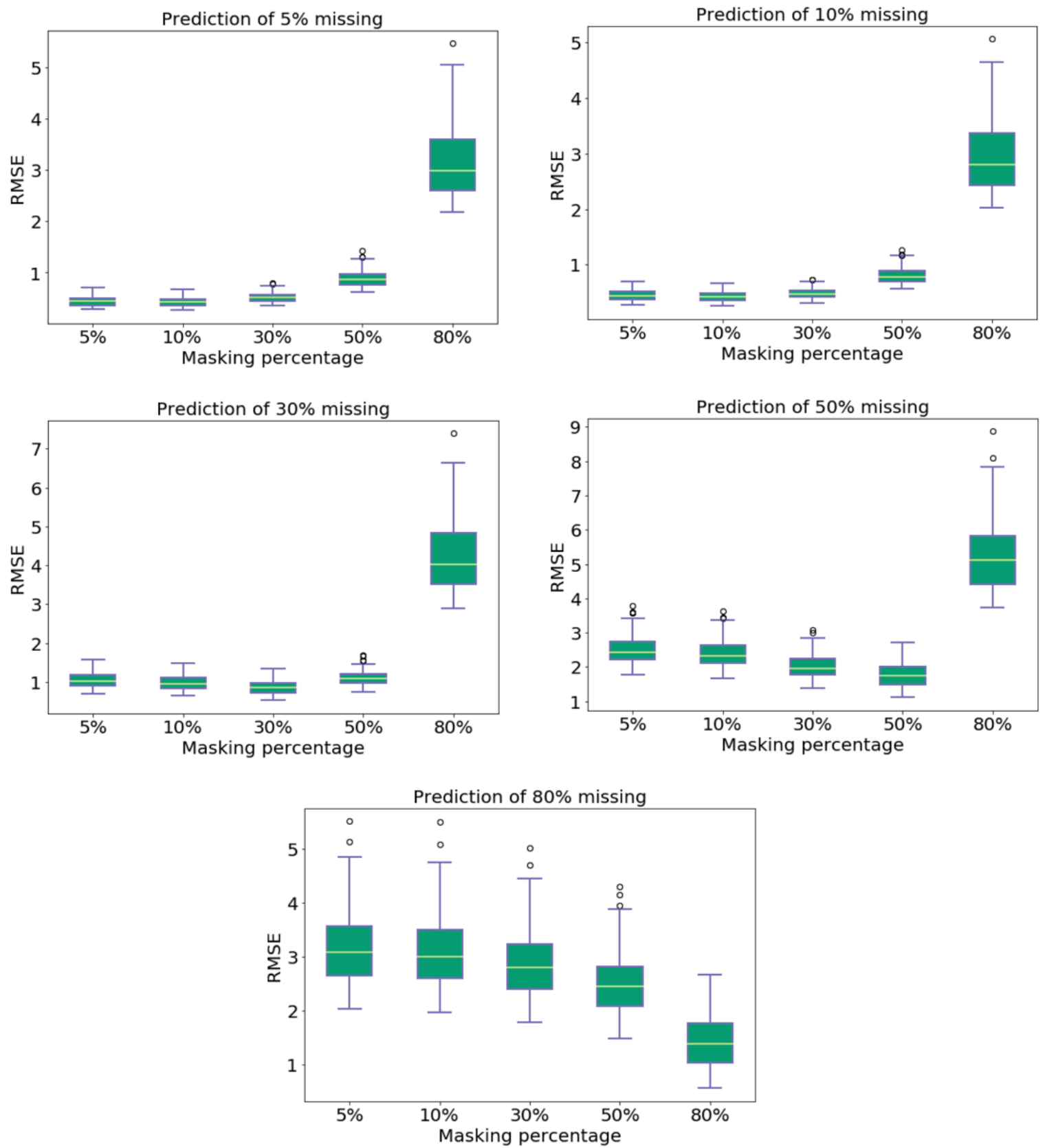

Figure 3. Effect of different masking percentages during training of denoising autoencoder with partial loss, measured in root mean square error (RMSE), for predicting at each of the 5 missing levels from top left to bottom right: $5 \%, 10 \%, 30 \%, 50 \%, 80 \%$ missing. 


\section{Optimal architecture for DAPL}

We evaluated twelve autoencoder architectures including three, five and seven layer networks with a range of layer-wise reduction factors. We found that reduction factor has a noticeable effect on imputation accuracies (Figure 4). The model complexity decreases with increasing layerwise reduction factor. In general, the trend is that the RMSE gets higher when the model becomes simpler. However, this is not strictly true. The RMSE trend drops slightly at the beginning for five layer autoencoders, and does not increase steeply within a certain range. This suggests that the optimal DAPL architecture is not the most complex one, and hence it is possible to reduce complexity without heavily degrading performance.

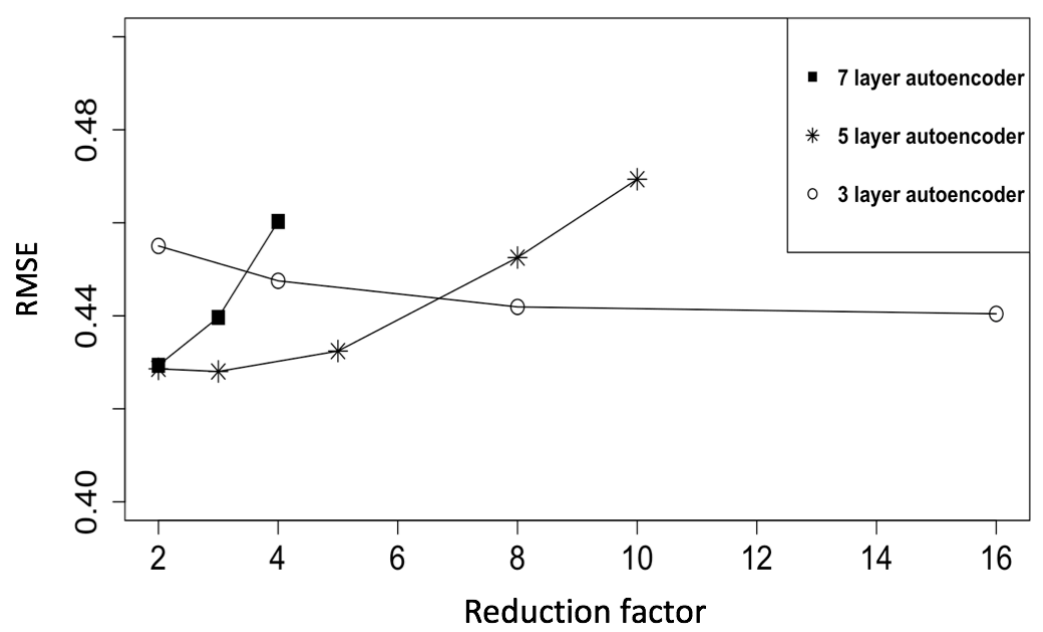

Figure 4. Results of autoencoder structural variations. Root mean square error (RMSE) is measured against layerwise size reduction factor for each of 3 layer, 5 layer, and 7 layer autoencoders.

\section{DAPL outperforms SVD for missing not at random}

Missing not at random cases include missing based on expression level where a random half of the genes with expression level at the lowest $5 \%$ or $10 \%$ are missing, and missing based 
on $\mathrm{GC}$ content where a random half of the genes with GC-content at the highest $5 \%$ or $10 \%$ are missing. Both were performed with an autoencoder with five hidden layers and bottleneck size of 800. For missing based on expression level cases, DAPL outperforms SVD, achieving the lowest RMSE among all methods in both missing percentages (Figure 5). For missing based on GCcontent cases, SVD has the lowest RMSE compared to other methods, followed by DAPL

(Figure 5).
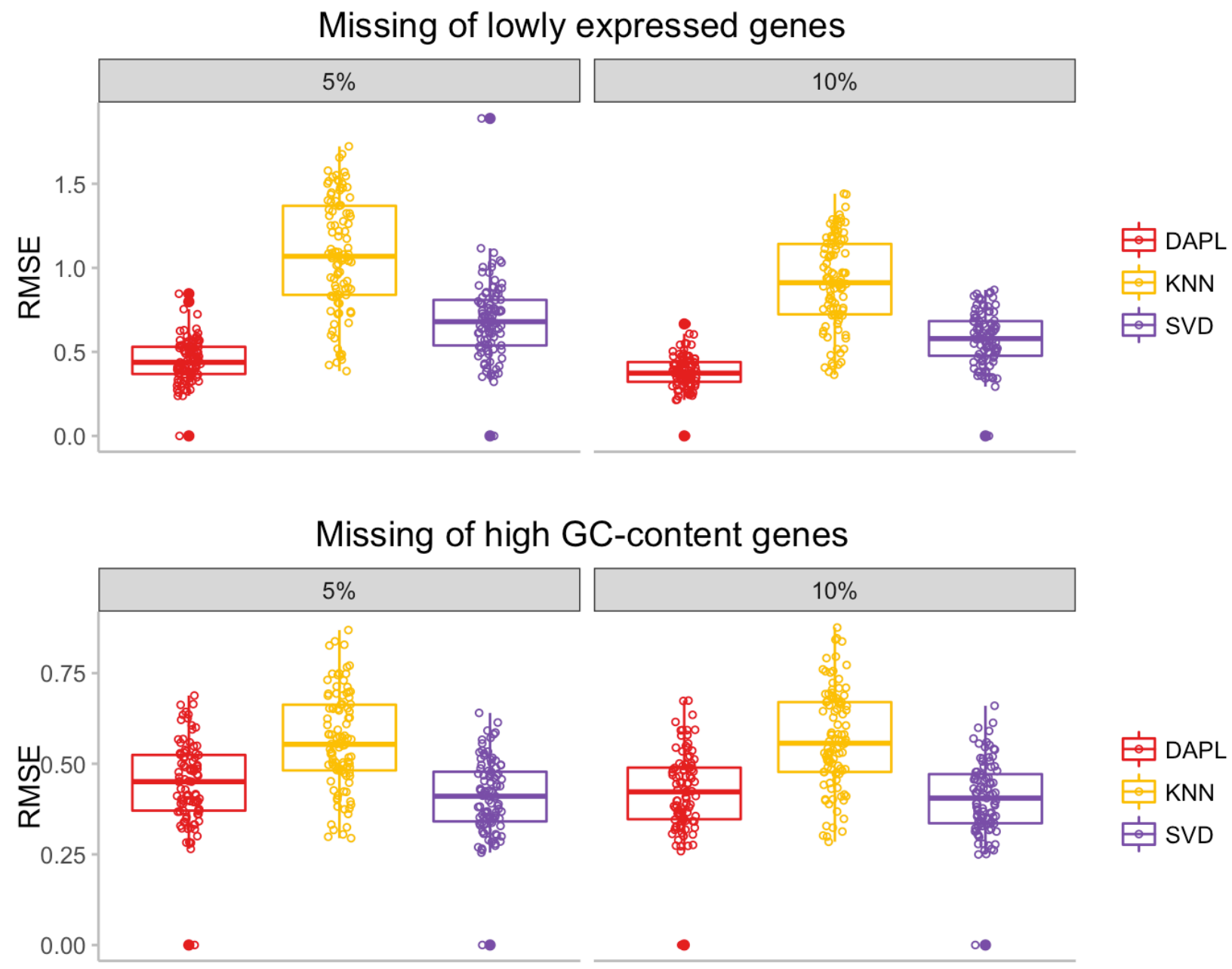

Figure 5. Comparisons of imputation methods measured in root mean square error (RMSE) for missing not at random cases. Top row from left to right: a random half of the genes with expression level at the lowest $5 \%$ and $10 \%$ are missing. Bottom row from left to right: a random half of the genes with GC-content at the highest $5 \%$ or $10 \%$ are missing. The method used from left to right: denoising autoencoder with partial loss (DAPL), 10 nearest neighbor method (KNN), and iterative singular value decomposition (SVD). 


\section{DAPL method performs similarly on DNA methylation data}

The method is validated on the TCGA pan-cancer DNA methylation data. This is to test whether DAPL is robust enough to work on data where the feature space is far greater than the sample size, which is a scenario in neural network-based methods that usually raises issues such as overfitting, and hence results in poor performances. On the methylation data, we see that due to increased data size, all of the imputation methods have higher RMSE than in the RNA sequencing data. On all simulation cases SVD still outperforms others, but DAPL shows similar or better result than KNN (Figure 6).

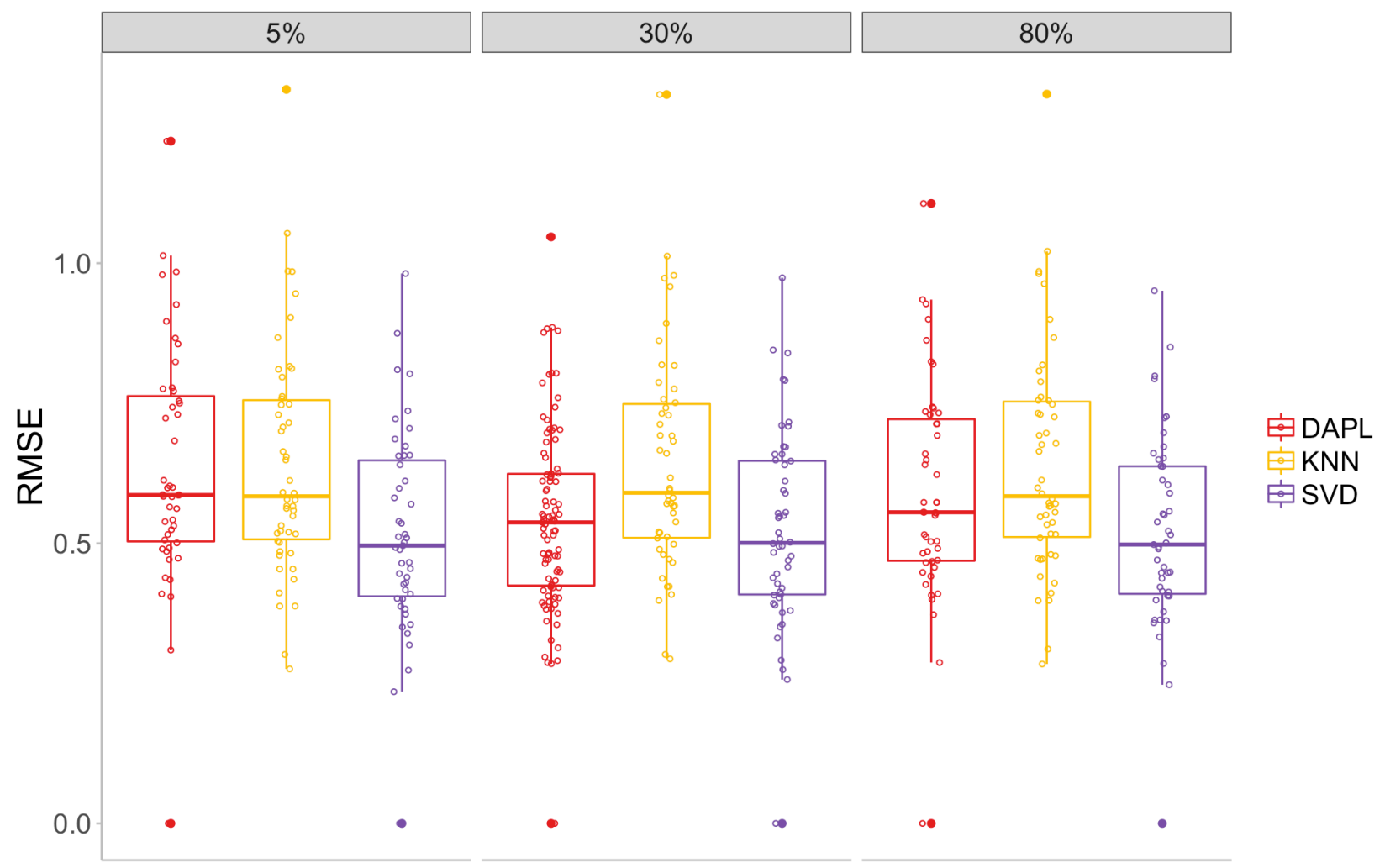

Figure 6. Comparisons of imputation methods measured in root mean square error (RMSE) for missing at random cases on DNA methylation data. From left to right: testing is done at $5 \%, 30 \%$, and $80 \%$ random missing. The method used from left to right: denoising autoencoder with partial loss (DAPL), 10 nearest neighbor method (KNN), and iterative singular value decomposition (SVD). 


\section{DAPL is the fastest method for evaluation.}

All computations were done on a 20 core cluster with Intel Xeon $2.40 \mathrm{GHz}$ CPUs. In terms of evaluation time, the KNN method took on average 8405 seconds to estimate missing values for a dataset with 100 samples and 16176 genes, with $5 \%$ of the entries missing, while SVD took 36911 seconds and DAPL took only 60 seconds, showing that DAPL is several orders of magnitude faster at evaluation time. 


\section{Conclusion}

We have described a new method, DAPL, on autoencoder neural network to impute missing data in large genomic datasets. Our results showed that with the modified partial loss it is possible to achieve significant improvement over standard denoising autoencoder. We have simulated multiple missing mechanisms including both missing-at-random cases across a range of missing percentages, and missing-not-at-random cases based on expression level and GCcontent. DAPL achieves similar imputation accuracies to the best performing competitor algorithm SVD. We also showed that imputation accuracy is not necessarily compromised by reducing model complexity. This is advantageous for working with large dataset such as in pancancer analysis (Cline, et al., 2013; Liu, et al., 2018).

DAPL has the benefit of reducing computational cost especially at evaluation time compared to traditional methods such as SVD and KNN. This is because an autoencoder model can be pre-trained and applied directly to any given new sample to impute missing values, while SVD and KNN methods are not model-based, and hence need to compute all values each time a new sample is given. DAPL provides a strong alternative to traditional methods for estimating missing values in large datasets. 


\section{Funding}

Research reported in this publication was supported by the National Institute of Dental \& Craniofacial Research (NIDCR) U01 DE025188, the National Institute of Biomedical Imaging and Bioengineering of the National Institutes of Health (NIBIB), R01 EB020527 and the National Cancer Institute (NCI) under U01 CA217851 and U01 CA199241. The content is solely the responsibility of the authors and does not necessarily represent the official views of the National Institutes of Health. The funders had no role in study design, data collection and analysis, decision to publish, or preparation of the manuscript. 


\section{References}

Aghdam, R., et al. The Ability of Different Imputation Methods to Preserve the Significant Genes and Pathways in Cancer. Genomics, Proteomics \& Bioinformatics 2017;15(6):396-404. Baghfalaki, T., Ganjali, M. and Berridge, D. Missing Value Imputation for RNA-Sequencing Data Using Statistical Models: A Comparative Study. Journal of Statistical Theory and Applications 2016;15. Ballard, D.H. Modular learning in neural networks. In, Proceedings of the sixth National conference on Artificial intelligence - Volume 1. Seattle, Washington: AAAI Press; 1987. p. 279284.

Beaulieu-Jones, B.K. and Moore, J.H. Missing Data Imputation in the Electronic Health Record Using Deeply Learned Autoencoders. Pac Symp Biocomput 2017;22:207-218. Byron, S.A., et al. Translating RNA sequencing into clinical diagnostics: opportunities and challenges. Nat Rev Genet 2016;17(5):257-271.

Cancer Genome Atlas Research, N., et al. The Cancer Genome Atlas Pan-Cancer analysis project. Nat Genet 2013;45(10):1113-1120.

Champion, M., et al. Module Analysis Captures Pancancer Genetically and Epigenetically Deregulated Cancer Driver Genes for Smoking and Antiviral Response. EBioMedicine 2018;27:156-166.

Chen, C.L., et al. Deep Learning in Label-free Cell Classification. Scientific Reports 2016;6:21471.

Chen, Y., et al. Gene expression inference with deep learning. Bioinformatics 2016;32(12):18321839.

Chen, Y.C., et al. Effects of GC bias in next-generation-sequencing data on de novo genome assembly. PLoS One 2013;8(4):e62856.

Cline, M.S., et al. Exploring TCGA Pan-Cancer Data at the UCSC Cancer Genomics Browser. Scientific Reports 2013;3:2652.

Conesa, A., et al. A survey of best practices for RNA-seq data analysis. Genome Biol 2016;17:13.

Faisal, S. and Tutz, G. Missing value imputation for gene expression data by tailored nearest neighbors. Stat Appl Genet Mol Biol 2017;16(2):95-106.

Gevaert, O., Tibshirani, R. and Plevritis, S.K. Pancancer analysis of DNA methylation-driven genes using MethylMix. Genome Biol 2015;16:17.

Hastie, T., et al. Imputingmissing data for gene expression arrays. 1999.

Jaques, N., et al. Multimodal autoencoder: A deep learning approach to filling in missing sensor data and enabling better mood prediction. 2018.

Kulis, M. and Esteller, M. DNA methylation and cancer. Adv Genet 2010;70:27-56.

Leung, M.K., et al. Deep learning of the tissue-regulated splicing code. Bioinformatics 2014;30(12):i121-129.

Libbrecht, M.W. and Noble, W.S. Machine learning applications in genetics and genomics. Nat Rev Genet 2015;16(6):321-332.

Litovkin, K., et al. DNA Methylation-Guided Prediction of Clinical Failure in High-Risk Prostate Cancer. PLoS One 2015;10(6):e0130651. 
Liu, B., et al. Deep neural networks for high dimension, low sample size data. In, Proceedings of the 26th International Joint Conference on Artificial Intelligence. Melbourne, Australia: AAAI Press; 2017. p. 2287-2293.

Liu, J., et al. An Integrated TCGA Pan-Cancer Clinical Data Resource to Drive High-Quality Survival Outcome Analytics. Cell 2018;173(2):400-416 e411. Min, S., Lee, B. and Yoon, S. Deep learning in bioinformatics. Brief Bioinform 2017;18(5):851869.

Sakurada, M. and Yairi, T. Anomaly Detection Using Autoencoders with Nonlinear Dimensionality Reduction. In, Proceedings of the MLSDA 2014 2nd Workshop on Machine Learning for Sensory Data Analysis. Gold Coast, Australia QLD, Australia: ACM; 2014. p. 4-11. Srivastava, N., et al. Dropout: a simple way to prevent neural networks from overfitting. $J$. Mach. Learn. Res. 2014;15(1):1929-1958.

Tomczak, K., Czerwinska, P. and Wiznerowicz, M. The Cancer Genome Atlas (TCGA): an immeasurable source of knowledge. Contemp Oncol (Pozn) 2015;19(1A):A68-77. Troyanskaya, O., et al. Missing value estimation methods for DNA microarrays. Bioinformatics 2001;17(6):520-525.

Vincent, P., et al. Extracting and composing robust features with denoising autoencoders. In, Proceedings of the 25th international conference on Machine learning. Helsinki, Finland: ACM; 2008. p. 1096-1103.

Vincent, P., et al. Stacked Denoising Autoencoders: Learning Useful Representations in a Deep Network with a Local Denoising Criterion. J. Mach. Learn. Res. 2010;11:3371-3408.

Wulsin, D.F., et al. Modeling electroencephalography waveforms with semi-supervised deep belief nets: fast classification and anomaly measurement. J Neural Eng 2011;8(3):036015. 